\title{
Group Duality and the Kubo-Martin Schwinger Condition. II $^{\star}$
}

\author{
Daniel Kastler ${ }^{\star \star}$ and Masamichi Takesaki \\ Department of Mathematics, University of California, Los Angeles, CA 90024, USA
}

\begin{abstract}
Let $\omega$ be an invariant state of the $C^{*}$-system $\{\mathfrak{U}, G, \alpha\}$ on a locally compact noncommutative group $G$. Assume further that $\omega$ is extremal $\tau$-invariant for an action $\tau$ of an amenable group $H$ which is $\omega$-asymptotically abelian and commutes with $\alpha$. Denoting by $F_{A B}, G_{A B}$ the corresponding two point functions, we give criteria for the fulfillment of the KMS condition with respect to some one parameter subgroup of the center of $G$ based on the existence of a closable map $T$ such that $T F_{A B}=G_{A B}$ for all $A, B \in \mathfrak{U}$. Closability is either in $L^{\infty}(G), B(G)$ or $C_{\infty}(G)$, according to clustering properties for $\tau$. The basic mathematical technique is the duality theory for noncompact, noncommutative locally compact groups.
\end{abstract}

\section{Introduction}

This paper continues the investigation of the relationship between group duality and the KMS condition undertaken in $[1,2]$ as a part of the general study of the mathematical structure of equilibrium states. Apart from their own interest for the description of temperature states, KMS states of $C^{*}$-dynamical systems appear as a natural testing ground for the generic features of dynamical automorphism groups (we recall that ground states are limiting cases of KMS states for a vanishing temperature, the KMS condition becoming positivity of the spectrum in the limit). Specifically we ask the question as to what generic properties of a $C^{*}$ dynamical system will cause its equilibrium states, defined, e.g., as the extremal invariant states, to exhibit the KMS structure. Although partial answers to these questions are known $[1,4,5]$ we feel that there should be more to say about distinctive features of dynamics as automorphism groups. In particular one is tempted to think that besides locality, a property of the quantal ergodicity type should be a generic feature of the interaction for infinite quantum systems,

* This work is supported in part by the National Science Foundation, Grant MCS 79-03041

$\star \star$ Permanent address: Centre de Physique Théorique, Luminy Case 907, F-13288 Marseille, Cedex 2, France 
possibly responsible, with locality, for the occurrence of the KMS structure. In order to get a handle on such questions it seems of interest to gather alternative mathematical formulations of the fact that a given state of a $C^{*}$-system is KMS for some one-parameter group of its isotropy subgroup. Such a criterium was given in [1] for the case of asymptotically abelian $C^{*}$-systems with an abelian automorphism group leaving the given state invariant. In the present paper we want to generalize some of the results in [1] to the technically more involved and possibly more interesting case of a noncommutative group. Namely, we look for conditions which enable us to find a one parameter subgroup of the preassigned automorphism group for which the state satisfies the KMS condition. In the abelian case, we handled this problem by making use of the Kampen-Pontryagin duality theorem. In the present paper, we shall instead appeal to the Tannaka-Tasuuma duality theorem for noncommutative groups. Except for this change in the basic tool, our investigation goes quite parallel to the abelian case.

\section{Framework and Notations Concerning Locally Compact Groups}

Let $G$ be a (non-commutative) locally compact group with left Haar measure $d s$ and modular function $\Delta$ :

$$
\left\{\begin{array}{l}
d(s t)=\Delta(t) d s \\
d\left(s^{-1}\right)=\Delta(s)^{-1} d s,
\end{array}\right.
$$

and set $L^{p}(G, d x)=L^{p}(G), 0<p \leqq \infty$. We consider the following diagrams of *-homomorphisms all of which but one are injections ${ }^{1}$

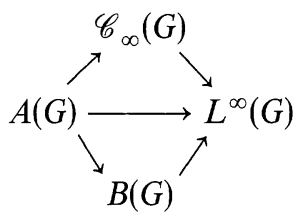

(b)

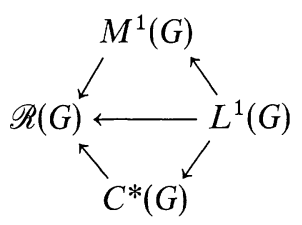

The algebras $(a)$ are injected into $L^{\infty}(G)$ as *-subalgebras of the latter endowed with its usual structure as a von Neumann algebra (i.e., with the pointwise product of functions and their complex conjugation $\left.{ }^{-}\right)$: These algebras are thus abelian. $C_{\infty}(G)$ is the algebra of continuous functions on $G$ vanishing at $\infty, B(G)$ the linear closure of functions of positive type on $G$ (the Fourier-Stielties algebra), the Fourier algebra $A(G)$ consists of the convolution products of two elements of $\mathscr{H}=L^{2}(G)$.

1 The map sending $C^{*}(G)$ into $\mathscr{R}(G)$ is injective if and only if $G$ is amenable. The involution is noted ${ }^{-}$for the algebras (a) and ${ }^{*}$ for the algebras (b) 
The algebras in the diagram (b) are noncommutative, they contain as a dense ${ }^{2}$ *-subalgebra the group algebra $L^{1}(G)$ with the convolution product

$$
(f * g)(s)=\int f(t) g\left(t^{-1} s\right) d t=\int f(s t) g\left(t^{-1}\right) d t
$$

and the involution

$$
f^{*}(s)=\Delta(s)^{-1} \overline{f\left(s^{-1}\right)} .
$$

$M^{1}(G)$ is the set of bounded regular measures on $G$ with the convolution of measures as a product, $C^{*}(G)$ the group $C^{*}$-algebra [the $C^{*}$-envelope of $L^{1}(G)$, and $\mathscr{R}(G)$ the weak closure of $L^{1}(G)$ in its regular representation $\lambda: \mathscr{R}(G)=\lambda\left(L^{1}(G)\right)^{\prime \prime}$ $=\lambda_{G}^{\prime \prime}$, where

$$
\begin{aligned}
& \left(\lambda_{s} \xi\right)(t)=\xi\left(s^{-1} t\right), \quad \xi \in L^{2}(G), \quad s, t \in G \\
& \lambda(f) \xi=f * \xi, \quad \xi \in L^{2}(G), \quad f \in L^{1}(G) .
\end{aligned}
$$

In addition to the left $G$-translations (4) we shall consider the right $G$-translations

$$
\varrho_{s}(\xi)(t)=\xi(t s)
$$

of arbitrary functions on $G$.

The above algebras appear in the following pairs $(\mathscr{A}, \mathscr{B})$ of algebras of different types (a), (b), one of which is the strong dual of the other ${ }^{3}$ :

$$
\left(A, \mathscr{R}=A^{*}\right), \quad\left(\mathscr{C}_{\infty}, M^{1}=\mathscr{C}_{\infty}^{*}\right), \quad\left(B=\left(C^{*}\right)^{*}, C^{*}\right), \quad\left(L^{\infty}=L^{1^{*}}, L^{1}\right) .
$$

The duality form for such pairs will be noted

$$
\langle a, b\rangle, \quad a \in \mathscr{A}, \quad b \in \mathscr{B},
$$

in particular, for $\mathscr{A}=L^{\infty}$ and $\mathscr{B}=L^{1} \quad 4$

$$
\langle a, b\rangle=\int a(s) b(s) d s=\left(b * a^{\natural}\right)(e)=\left(b^{\wedge} * a\right)(e), \quad a \in \mathscr{A}, \quad b \in \mathscr{B} .
$$

The definition of $A$ as $L^{2} * L^{2}$ follows from the more natural definition as the predual of the von Neumann algebra $\mathscr{R}$ [or equivalently as the set of bounded linear forms on $L^{1}(G)$ or $C^{*}(G)$ normal for the regular representation $\left.\lambda\right]$ : since $\mathscr{R}$ is standard each of its normal states is a vector state, it follows thus by polarization that $A$ consists of the following "dyads" $\mid \eta)\left(\xi \mid:^{5}\right.$

$$
\langle| \eta)\left(\xi|, b\rangle=(\xi \mid b \eta), \quad \eta, \xi \in L^{2}, \quad b \in \mathscr{R} .\right.
$$

For $a=\lambda(u), u \in L^{1}$, this reads

$$
\begin{aligned}
\langle| \eta)(\xi|, \lambda(u)\rangle & =\int \bar{\xi}(s) u(t) \eta\left(t^{-1} s\right) d s d t \\
& =\int\left(\bar{\xi} * \eta^{\urcorner}\right)(t) u(t) d t,
\end{aligned}
$$

2 Either in their weak or in the weak * topology

3 We shall henceforth omit in the symbols (a), (b) the explicit mention of the group $G$ given once and for all

4 See below for the definition of ${ }^{2}$ and ${ }^{\wedge}$

$5(f \mid g)$ denotes the scalar product in $\mathscr{H}=L^{2}$ considered according to the physicist's convention as linear in $g$ 
where $f^{\sim}$ denotes the function

$$
f^{\curlyvee}(t)=f\left(t^{-1}\right), \quad t \in G .
$$

We note that the map (12) is an involutory isomorphism of each of the algebras $\mathscr{A}$ of type (a). We denote by ${ }^{\wedge}$ the involutory antiisomorphism of the corresponding algebra $\mathscr{B}$ obtained by transposition

$$
\left\langle a, b^{\dagger}\right\rangle=\langle\hat{a}, b\rangle, \quad a \in \mathscr{A}, \quad b \in \mathscr{B} .
$$

We have, for $\mathscr{B}=L^{1}$,

$$
f^{\wedge}(s)=\Delta(s)^{-1} f\left(s^{-1}\right), \quad f \in L^{1}, \quad s \in G .
$$

We shall also consider on the algebras $\mathscr{B}$ the operation ${ }^{-}$given by

$$
\bar{b}=\left(b^{*}\right)^{\wedge}=\left(b^{\wedge}\right)^{*}, \quad b \in \mathscr{B},
$$

coinciding on $L^{1}$ with complex conjugation.

We recall the known fact that for each of the algebras $\mathscr{A}, \mathscr{B}$ in (a), (b) the involution is continuous and the product separately continuous for the topology $\sigma(\mathscr{A}, \mathscr{B})$, respectively $\sigma(\mathscr{B}, \mathscr{A})$. The $G$-translations (4), (6) leave the algebras $\mathscr{A}$ invariant and are $\sigma(\mathscr{A}, \mathscr{B})$-continuous, so that we may define

$$
\left\{\begin{array}{l}
\left\langle\lambda_{s} a, b\right\rangle=\left\langle a, \lambda_{s^{-1}} b\right\rangle \\
\left\langle\lambda_{s}^{\prime} a, b\right\rangle=\left\langle a, \varrho_{s^{-1}} b\right\rangle
\end{array} \quad s \in G, \quad a \in \mathscr{A}, \quad b \in \mathscr{B},\right.
$$

whereby $\lambda_{s}$ on $\mathscr{B}=L^{1}$ is consistent with (4) whilst $\lambda_{s}^{\prime}=\Delta(s) \varrho_{s}$.

In the proofs to follow we shall treat in a uniform manner the pairs of algebras $(\mathscr{A}, \mathscr{B})$ in $(7)$. It is therefore appropriate to cast our assumptions in the following abstract manner subsuming the above facts.

Definition 1. We say that two Banach $*$ algebras $\mathscr{A}$ and $\mathscr{B}$ are in duality if the following conditions hold:

(i) $\mathscr{A}=\mathscr{B}^{*}$ or $\mathscr{B}=\mathscr{A}^{*}$;

(ii) for each $a_{0} \in \mathscr{A}$, the maps $a \in \mathscr{A} \rightarrow a_{0} a \in \mathscr{A}$ and $a \in \mathscr{A} \rightarrow a a_{0} \in \mathscr{A}$ are both $\sigma(\mathscr{A}, \mathscr{B})$-continuous;

(ii') for each $b_{0} \in \mathscr{B}$, the maps $b \in \mathscr{B} \rightarrow b_{0} b \in \mathscr{B}$ and $b \in \mathscr{B} \rightarrow b b_{0} \in \mathscr{B}$ are both $\sigma(\mathscr{B}, \mathscr{A})$-continuous;

(iii) the antilinear involutions ${ }^{-}$of $\mathscr{A}$, and $*$ of $\mathscr{B}$, are respectively $\sigma(\mathscr{A}, \mathscr{B})$ - and $\sigma(\mathscr{B}, \mathscr{A})$-continuous.

Assumptions. With the notation introduced above for the locally compact group $G$, we assume the existence of a diagram

$$
\begin{gathered}
A(G) \stackrel{\pi_{*}}{\longrightarrow} \mathscr{A} \stackrel{\hat{\pi}}{\longrightarrow} L^{\infty}(G) \\
\mathscr{R}(G) \stackrel{\pi}{\longleftarrow} \mathscr{B} \stackrel{\hat{\pi}_{*}}{\longleftarrow} L^{1}(G)
\end{gathered}
$$

fulfilling the following conditions:

I) $\mathscr{A}$ and $\mathscr{B}$ are Banach $*$ algebras in duality.

II) $\pi, \pi_{*}$, and $\hat{\pi}, \hat{\pi}_{*}$ are pairs of adjoint maps; and $\pi_{*}, \hat{\pi}, \hat{\pi}_{*}$ are *-monomorphisms, whilst $\pi$ is a $*$-homomorphism. 
III) $\hat{\pi}^{\circ} \pi_{*}$ is the canonical embedding of $A$ into $L^{\infty} ; \pi \circ \hat{\pi}_{*}=\lambda$, the regular representation (5) of $L^{1}$.

IV) The group $G$ acts on $\mathscr{A}$ bilaterally as follows

$$
\left\{\begin{array}{l}
\hat{\pi}\left(\lambda_{s}(a)\right)(t)=\hat{\pi}(a)\left(s^{-1} t\right) \\
\hat{\pi}\left(\varrho_{s}(a)\right)(t)=\hat{\pi}(a)(t s)
\end{array}, \quad s, t \in G, \quad a \in \mathscr{A} .\right.
$$

V) $\mathscr{A}$ possesses a $\sigma(\mathscr{A}, \mathscr{B})$-continuous involutory automorphism ${ }^{2}$, thus

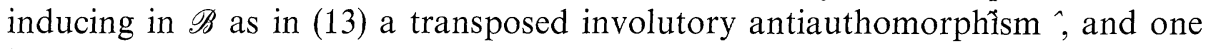
has

$$
\left\{\begin{array}{c}
\hat{\pi}\left(a^{\natural}\right)=\hat{\pi}(a)^{\nu}, \quad a \in \mathscr{A} \\
\hat{\pi}_{*}\left(b^{\wedge}\right)=\hat{\pi}_{*}(b)^{\wedge}, \quad b \in \mathscr{B},
\end{array}\right.
$$

where ${ }^{\wedge}$ and ${ }^{\wedge}$ on the right hand side are the involutions (12) and (14) of $L^{\infty}$, respectively $L^{1}$.

For more clarity we shall use different notations for the products in $\mathscr{A}$ and $\mathscr{B}$ : the former will be denoted $a_{1} \cdot a_{2}$ (corresponding via $\hat{\pi}$ to the pointwise product of functions), the latter $b_{1} b_{2}$ (coinciding on $L^{1}$ with the involution).

Property (ii) allows us to define as follows an action of $\mathscr{A}$ on $\mathscr{B}$

$$
\left\langle a_{1} \cdot a_{2}, b\right\rangle=\left\langle a_{1}, a_{2} \cdot b\right\rangle, \quad a_{1}, a_{2} \in \mathscr{A}, \quad b \in \mathscr{B} .
$$

Property (ii') on the other hand yields as follows a right and a left action of $\mathscr{B}$ on $\mathscr{A}$

$$
\left\langle a, b_{1} b_{2}\right\rangle=\left\langle a b_{2}^{\nu}, b_{1}\right\rangle=\left\langle b_{1}^{\hat{1}} a, b_{2}\right\rangle, \quad a \in \mathscr{A}, \quad b_{1}, b_{2} \in \mathscr{B} .
$$

We end up this section with three lemmas stating intuitive technical facts needed in the sequel.

Lemma 1. For $f \in L^{1}$ we have

$$
\left\{\begin{array}{l}
\lambda_{f}(a)=\int f(s) \lambda_{s}(a) d s=\hat{\pi}_{*}\left(f^{\wedge}\right)^{\wedge} a \\
\varrho_{f}(a)=\int f(s) \varrho_{s}(a) d s=a \hat{\pi}_{*}(f)^{\nu} .
\end{array}\right.
$$

Proof. We have, for $g \in L^{1}$

$$
\begin{aligned}
\left\langle\lambda_{f}(a), \hat{\pi}_{*}(g)\right\rangle & =\int f(s)\left\langle\lambda_{s}(a), \hat{\pi}_{*}(g)\right\rangle d s \\
& =\int f(s)\left\langle\hat{\pi}\left(\lambda_{s}(a)\right), g\right\rangle d s \\
& =\iint f(s) g(t) \hat{\pi}\left(\lambda_{s}(a)\right)(t) d s d t \\
& =\iint f(s) g(t) \hat{\pi}(a)\left(s^{-1} t\right) d s d t \\
& =\iint f(s) g(s t) \hat{\pi}(a)(t) d s d t=\left\langle\hat{\pi}(a), f^{\wedge} * g\right\rangle \\
& =\left\langle a, \hat{\pi}_{*}\left(f^{\wedge} * g\right)\right\rangle=\left\langle a, \hat{\pi}_{*}\left(f^{\wedge}\right) \hat{\pi}_{*}(g)\right\rangle \\
& =\left\langle\pi_{*}\left(f^{\wedge}\right)^{\wedge} a, \hat{\pi}_{*}(g)\right\rangle .
\end{aligned}
$$

The second relation in (22) is checked similarly.

Lemma 2. For $b \in \mathscr{B}, \xi, \eta \in \mathscr{H}$ we have

$$
\left\{\begin{array}{l}
b^{\wedge} \pi_{*}\{\mid \xi)(\eta \mid\}=\pi_{*}\{\mid \xi)\left(\pi\left(b^{*}\right) \eta \mid\right\} \\
\pi_{*}\{\mid \xi)(\eta \mid\} b^{2}=\pi_{*}\{\mid \pi(b) \xi)(\eta \mid\} .
\end{array}\right.
$$


Proof. We have, for $b_{1} \in \mathscr{B}$

$$
\begin{aligned}
\left\langle b^{\wedge} \pi_{*}\{\mid \xi)(\eta \mid\}, b_{1}\right\rangle & \left.=\left\langle\pi_{*}\{\mid \xi)(\eta \mid\}, b b_{1}\right\rangle=\langle| \xi\right)\left(\eta\left|, \pi\left(b b_{1}\right)\right\rangle\right. \\
& =\left(\eta \mid \pi\left(b b_{1}\right) \xi\right)=\left(\pi\left(b^{*}\right) \eta \mid \pi\left(b_{1}\right) \xi\right) \\
& =\langle| \xi)\left(\pi\left(b^{*}\right) \eta\left|, \pi\left(b_{1}\right)\right\rangle\right. \\
& =\left\langle\pi_{*}\{\mid \xi)\left(\pi\left(b^{*} \eta\right) \mid\right\}, b_{1}\right\rangle .
\end{aligned}
$$

The proof of the second relation (24) is analogous.

Let $i$ be the natural imbedding of $\mathscr{H} \cap L^{\infty}(G)$ into $L^{\infty}(G)$. When a function $f \in \mathscr{H}$ gives rise to a bounded operator as the convolution from the left: $g \in \mathscr{H} \mapsto f * g$, we denote the corresponding operator by $\lambda(f)$. Of course, $\lambda(f)$ belongs to $\mathscr{R}(G)$.

Lemma 3. Denote by $\mathscr{H}_{0}$ the subset of $\mathscr{H}$ consisting of the convolution products $f * g, f, g$ compactly supported continuous functions on $G$. We have, for $b \in \mathscr{B}$ and $f_{0} \in \mathscr{H}$

$$
i\left(\pi\left(b^{\wedge}\right) f_{0}\right)=\hat{\pi}\left(b^{\wedge} a_{0}\right),
$$

where $a_{0}$ is the element of $\mathscr{A}$ such that $i\left(f_{0}\right)=\hat{\pi}\left(a_{0}\right)$ and

$$
\lambda\left(\pi(b) f_{0}\right)=\pi(b) \lambda\left(f_{0}\right) .
$$

Proof. Let $f_{0}=\bar{\eta} * \xi^{\nu}, \eta, \xi \in \mathscr{C}_{K}(G)$. We have according to (11)

$$
\left(\hat{\pi} \circ \pi_{*}\right)\{\mid \xi)(\eta \mid\}=i(\bar{\eta} * \xi),
$$

thus

$$
a_{0}=\pi_{*}\{\mid \xi)(\eta \mid\}
$$

hence, by (24)

$$
b \hat{a} a_{0}=\pi_{*}\{\mid \xi)\left(\pi\left(b^{*}\right) \eta \mid\right\}
$$

thus

$$
\left.\hat{\pi}\left(b^{\hat{a}} a_{0}\right)=\hat{\pi} \circ \pi_{*}\{\mid \xi)\left(\pi\left(b^{*}\right) \eta \mid\right\}=i \overline{\left(\pi\left(b^{*}\right) \eta\right.} * \xi\right)=i\left(\pi\left(b^{\wedge}\right) f_{0}\right) .
$$

We proved (26). Now, for any $x \in \mathscr{R}(G), g \in \mathscr{H}_{0}$, we have

$$
\left(x f_{0}\right) * g=\lambda^{\prime}(g) x f=x \lambda^{\prime}(g) f=x\left(f_{0} * g\right),
$$

since $\lambda^{\prime}(g) \in \mathscr{R}(G)^{\prime}$, so that $x f_{0}$ is left bounded and $\lambda\left(x f_{0}\right)=x \lambda\left(f_{0}\right)$. Thus (27) follows.

\section{The Operator T: A Duality Theorem}

In this section $T$ denotes a $\sigma(\mathscr{A}, \mathscr{B})$-closed linear operator on $\mathscr{A}$ with $\sigma(\mathscr{A}, \mathscr{B})$ dense domain $\mathscr{D}$. We consider the properties

$\left(T_{1}\right) \varrho_{s}(\mathscr{D}) \subset \mathscr{D}$; and $T \circ \varrho_{s}=\varrho_{s} \circ T$ for all $s \in G$

$\left(T_{1}^{\prime}\right) \lambda_{s}(\mathscr{D}) \subset \mathscr{D}$; and $T \circ \lambda_{s}=\lambda_{s} \circ T$ for all $s \in G$

$\left(T_{2}\right) \mathscr{D} \cdot \mathscr{D} \subset \mathscr{D}$; and $T\left(a_{1} a_{2}\right)=T\left(a_{1}\right) T\left(a_{2}\right)$ for all $a_{1}, a_{2} \in \mathscr{D}$.

In what follows we seek to characterize such operators $T$. 
Lemma 4. Let $T$ be $\sigma(\mathscr{A}, \mathscr{B})$-closed with $\sigma(\mathscr{A}, \mathscr{B})$-dense domain $\mathscr{D}$ and denote $T^{\prime}$, with domain $\mathscr{D}^{\prime}$, the adjoint of $T$ on $\mathscr{B}$ if $\left(T_{1}\right)$ holds one has

(i) $\mathscr{D} \mathscr{B}^{2} \subset \mathscr{D}$; and $T\left(a_{0} b^{2}\right)=\left(T a_{0}\right) b^{2}, a_{0} \in \mathscr{D}, b \in \mathscr{B}$;

(ii) $\mathscr{D}^{\prime} \mathscr{B} \subset \mathscr{D}^{\prime}$; and $T^{\prime}\left(b_{0} b\right)=\left(T^{\prime} b_{0}\right) b, b_{0} \equiv \mathscr{D}^{\prime}, b=\mathscr{B}$; if $\left(T_{1}^{\prime}\right)$ holds one has

(i') $\mathscr{B} \mathscr{D} \subset \mathscr{D}$; and $T\left(b^{\wedge} a_{0}\right)=b^{\wedge}\left(T a_{0}\right), a \in \mathscr{D}, b \in \mathscr{B}$;

(ii') $\mathscr{B} \mathscr{D}^{\prime} \subset \mathscr{D}^{\prime}$ and $T^{\prime}\left(b b_{0}\right)=b\left(T^{\prime} b_{0}\right), b_{0} \in \mathscr{D}^{\prime}, b \in \mathscr{B}$;

if $\left(T_{1}\right)$ and $\left(T_{1}^{\prime}\right)$ both hold one has

(ii") $T^{\prime}\left(b_{0} b_{1}\right)=b_{0}\left(T^{\prime} b_{1}\right)=\left(T^{\prime} b_{0}\right) b_{1}, b_{0}, b_{1} \in \mathscr{B}^{\prime}$;

(iii) $\mathscr{A} \mathscr{D}^{\prime \prime} \subset \mathscr{D}$; and $T\left(a b_{0}^{\llcorner}\right)=a\left(T^{\prime} b_{0}\right)^{2}, b_{0} \in \mathscr{D}^{\prime}, a \in \mathscr{A}$;

(iii') $\mathscr{D}^{\prime \wedge} \mathscr{A} \subset \mathscr{D}$; and $T\left(b_{0} a\right)=\left(T^{\prime} b_{0}\right)^{\wedge} a, b_{0} \in \mathscr{D}^{\prime}, a \in \mathscr{A}$;

if $\left(T_{2}\right)$ holds one has

(iv) $(T \mathscr{D}) \cdot \mathscr{D}^{\prime} \subset \mathscr{D}^{\prime}$; and $T^{\prime}\left(\left(T a_{0}\right) \cdot b_{0}\right)=a_{0} \cdot\left(T^{\prime} b_{0}\right), a_{0} \in \mathscr{D}, b_{0} \in \mathscr{D}^{\prime}$.

Proof. (i) and (i'): let $a_{0} \in \mathscr{D}, b \in \mathscr{B}$. By the closedness of $T$, and $\left(T_{1}\right)$, respectively $\left(T_{1}^{\prime}\right)$, one has for $f \in L^{1}$ that $\varrho_{f} \mathscr{D} \subset \mathscr{D}, T \circ \varrho_{f}=\varrho_{f} \circ T$, respectively $\lambda_{f} \mathscr{D} \subset \mathscr{D}, T \circ \lambda_{f}=\lambda_{f} \circ T$. But Lemma 1 tells us that $\varrho_{f}$ and $\lambda_{f}$ are the right, respectively, left, multiplication by $\hat{\pi}_{*}(f)^{\sim}$, respectively $\hat{\pi}_{*}\left(f^{\wedge}\right)^{\wedge}$. Since the latter are dense in $\mathscr{B}, b$ can be approximated by sequences $\hat{\pi}_{*}\left(f_{n}\right)^{2}$, respectively $\hat{\pi}_{*}\left(f_{n}^{\wedge}\right)^{\wedge}, f_{n} \in L^{1}$, yielding

$$
a_{0} \hat{\pi}_{*}\left(f_{n}\right)^{\nu} \underset{n=\infty}{\longrightarrow} a_{0} b ; \quad T\left(a_{0} \hat{\pi}_{*}\left(f_{n}\right)^{n}\right)=\left(T a_{0}\right) \hat{\pi}_{*}\left(f_{n}\right)^{\wedge} \underset{n=\infty}{\longrightarrow}\left(T a_{0}\right) b_{0},
$$

respectively

$$
\hat{\pi}_{*}\left(f_{n}^{\wedge}\right)^{\wedge} a \underset{n=\infty}{\longrightarrow} b a_{0} ; \quad T\left(\hat{\pi}_{*}\left(f_{n}^{\wedge}\right)^{\wedge} a_{0}\right)=\hat{\pi}_{*}\left(f_{n}^{\wedge}\right)^{\wedge} T a_{0} \underset{n=\infty}{\longrightarrow} b\left(T a_{0}\right),
$$

whence (i), respectively (i'), by the closedness of $T$.

(ii) and (ii'): we have, for $a_{0} \in \mathscr{D}, b_{0} \in \mathscr{D}^{\prime}$ and $b \in \mathscr{D}$, using (i), respectively (i'):

$$
\begin{aligned}
\left\langle T a_{0}, b_{0} b\right\rangle & =\left\langle\left(T a_{0}\right) b^{\nu}, b_{0}\right\rangle=\left\langle T\left(a_{0} b^{\nu}\right), b_{0}\right\rangle=\left\langle a_{0} b^{2}, T^{\prime} b_{0}\right\rangle \\
& =\left\langle a_{0},\left(T^{\prime} b_{0}\right) b\right\rangle,
\end{aligned}
$$

respectively

$$
\begin{aligned}
\left\langle T a_{0}, b b_{0}\right\rangle & =\left\langle b^{\wedge}\left(T a_{0}\right), b_{0}\right\rangle=\left\langle T\left(b^{\wedge} a_{0}\right), b_{0}\right\rangle=\left\langle b^{\wedge} a_{0}, T^{\prime} b_{0}\right\rangle \\
& =\left\langle a_{0}, b\left(T^{\prime} b_{0}\right)\right\rangle,
\end{aligned}
$$

(ii") : follows from (ii) and (ii').

(iii) and (iii'): we have, for $a \in \mathscr{A}$ and $b_{0}, b_{1} \in \mathscr{D}^{\prime}$, using (ii')

$$
\left\langle a b_{0}^{\circ}, T^{\prime} b_{1}\right\rangle=\left\langle a,\left(T^{\prime} b_{1}\right) b_{0}\right\rangle=\left\langle a, b_{1}\left(T^{\prime} b_{0}\right)\right\rangle=\left\langle a\left(T^{\prime} b_{0}\right)^{2}, b_{1}\right\rangle
$$

and

$$
\left\langle b_{0} a, T^{\prime} b_{1}\right\rangle=\left\langle a, b_{0}\left(T^{\prime} b_{1}\right)\right\rangle=\left\langle a,\left(T^{\prime} b_{0}\right) b_{1}\right\rangle=\left\langle\left(T^{\prime} b_{0}\right)^{\wedge} a, b_{1}\right\rangle .
$$

(iv): we have by $\left(T_{2}\right)$, for $a_{1}, a_{2} \in \mathscr{D}$ and $b_{0} \in \mathscr{D}^{\prime}$

$$
\begin{aligned}
\left\langle T a_{1},\left(T a_{2}\right) \cdot b_{0}\right\rangle & =\left\langle\left(T a_{1}\right) \cdot\left(T a_{2}\right), b_{0}\right\rangle=\left\langle T\left(a_{1} \cdot a_{2}\right), b_{0}\right\rangle \\
& =\left\langle a_{1} \cdot a_{2}, T^{\prime} b_{0}\right\rangle=\left\langle a_{1}, a_{2} \cdot\left(T^{\prime} b_{0}\right)\right\rangle .
\end{aligned}
$$


Lemma 5. Let $T$ be as in Lemma 4 and assume $\left(T_{1}\right),\left(T_{1}^{\prime}\right),\left(T_{2}\right)$. Let further $\mathscr{D}_{00} \subset \mathscr{D}_{0}$ and $\mathscr{D}_{00}^{*} \subset \mathscr{D}_{0}$ be defined as

$$
\begin{array}{rlrl}
\mathscr{D}_{0} & =\left[\pi\left(\mathscr{D}^{\prime}\right) \mathscr{H}\right], & \mathscr{D}_{00} & =\left[\pi\left(\mathscr{D}^{\prime \wedge}\right) \mathscr{H}_{0}\right], \\
\mathscr{D}_{0}^{*}=\left[\pi\left(\mathscr{D}^{\prime \prime}\right)^{*} \mathscr{H}\right] & =\left[\pi\left(\overline{\mathscr{D}}^{\prime}\right) \mathscr{H}\right], & \mathscr{D}_{00}^{*}=\left[\pi\left(\mathscr{D}^{\prime \prime}\right)^{*} \mathscr{H}_{0}\right]=\left[\pi\left(\overline{\mathscr{D}}^{\prime}\right) \mathscr{H}_{0}\right],
\end{array}
$$

where [] indicates a linear closure and $\mathscr{H}_{0}$ is as in Lemma 3. There are mutually adjoint closed operators $\mathbb{T}, \mathbb{T}^{*}$ on $\mathscr{H}$, with domain $\mathscr{D}_{\mathbb{T}} \supset \mathscr{D}_{0}$, respectively $\mathscr{D}_{\mathbb{T}^{*}} \supset \mathscr{D}_{0}^{*}$, such that

$$
\begin{gathered}
\mathbb{T} \pi\left(b_{0}\right) f=\pi\left\{\left(T^{\prime} b_{0}\right)^{\wedge}\right\} f, \\
\left\{\begin{array}{l}
\mathbb{T}^{*} \pi\left(b_{0}\right)^{*} f=\pi\left\{\left(T^{\prime} b_{0}\right)^{\wedge}\right\}^{*} f \quad \text { or } \\
\mathbb{T}^{*}\left(\pi\left(\bar{b}_{0}\right) f\right)=\pi \overline{\left(T^{\prime} b_{0}\right)} f
\end{array}, \quad f \in \mathscr{H}, \quad b_{0} \in \mathscr{D}^{\prime} .\right.
\end{gathered}
$$

Furthermore $\mathscr{D}_{00}$ and $\mathscr{D}_{00}^{*}$ are cores of $\mathbb{T}$, respectively $\mathbb{T}^{*}$ on which $\mathbb{T}$ and $\mathbb{T}^{*}$ are related as follows with $T$, respectively $T^{\prime}$ : we have

$$
\left\{\begin{array}{l}
i\left(\mathscr{D}_{00}\right) \subset \hat{\pi}(\mathscr{D}) \quad \text { and } \\
i(\mathbb{T} f)=\hat{\pi}(T a), \quad f \in \mathscr{D}_{00}, \quad a \in \mathscr{A} \quad \text { such that } \quad \hat{\pi}(a)=i(f)
\end{array}\right.
$$

and

$$
\left\{\begin{array}{l}
\lambda\left(\mathscr{D}_{00}^{*}\right) \subset \pi\left(\overline{\mathscr{D}}^{\prime}\right) \quad \text { and } \\
\lambda\left(\mathbb{T}^{*} \pi(b) f\right)=\pi\left(\bar{T}^{\prime} b\right) \lambda(f), \quad f \in \mathscr{H}_{0}, \quad b \in \overline{\mathscr{D}}^{\prime},
\end{array}\right.
$$

where $\bar{T}^{\prime}$ is defined by $\bar{T}^{\prime} \bar{k}=\overline{T^{\prime} k}, k \in \mathscr{D}^{\prime}$. Finally $\mathbb{T}$ is affiliated with the center of $\mathscr{R}$ and one has, with $W: L^{2}(G \times G) \rightarrow L^{2}(G \times G)$ defined as in the appendix

$$
W(\mathbb{T} \otimes \mathbb{T})=(\mathbb{T} \otimes \mathbb{1}) W .
$$

Proof. We first show that $\mathscr{D}_{0}$ and $\mathscr{D}_{0}^{*}$ are dense in $\mathscr{H}:$ if $g \in \mathscr{H}$ is orthogonal to $\mathscr{D}_{0}$ (respectively $\mathscr{D}_{0}^{*}$ ) we have indeed, for all $f \in \mathscr{H}$ and $b_{0} \in \mathscr{D}^{\prime}$ :

$$
\left\{\begin{array}{l}
\left.0=\left(g, \pi\left(b_{0}\right) f\right)=\langle| f\right)\left(g\left|, \pi\left(b_{0}\right)\right\rangle=\langle| f\right)\left(\left.g\right|^{\llcorner}, \pi\left(b_{0}\right)\right\rangle, \\
\left.\left(\text { respectively } 0=\left(\pi\left(\bar{b}_{0}\right) f \mid g\right)=\langle| g\right)\left(f\left|, b_{0}\right\rangle=\langle| g\right)\left(\left.f\right|^{\nu}, b_{0}\right\rangle\right) .
\end{array}\right.
$$

Thus, by the $\sigma(\mathscr{B}, \mathscr{A})$ density of $\mathscr{D}^{\prime}$ in $\left.\mathscr{B}, \mid f\right)\left.\left(\left.g\right|^{2}=0\right.$ (respectively $\left.\mid g\right)(f)\right|^{\swarrow}=0$ ) for all $f \in \mathscr{H}$, thus $g=0$.

Now we notice that, for $f, f^{\prime} \in \mathscr{H}$ and $b_{0}, b_{1} \in \mathscr{D}^{\prime}$

$$
\left(\pi\left\{\left(T^{\prime} b_{0}\right)^{\wedge}\right\} f \mid \pi\left(b_{1}\right)^{*} f^{\prime}\right)=\left(\pi\left(b_{0}^{\wedge}\right) f \mid \pi\left\{\left(T^{\prime} b_{1}\right\}^{*} f^{\prime}\right)\right.
$$

as immediately follows from Lemma 4 : this shows that the relations

$$
\left\{\begin{array}{l}
T_{0} \pi\left(b_{0}\right) f=\pi\left\{\left(T^{\prime} b_{0}\right)^{\wedge}\right\} f \\
S_{0} \pi\left(b_{0}\right)^{*} f=\pi\left\{\left(T^{\prime} b_{0}\right)^{\wedge}\right\}^{*} f
\end{array}, \quad f \in \mathscr{H}, \quad b_{0} \in \mathscr{D}^{\prime}\right.
$$

coherently define closable linear operators $T_{0}, S_{0}$ with domains $\mathscr{D}_{0}$, respectively $\mathscr{D}_{0}^{*}$, such that $S_{0} \subset T_{0}^{*}, T_{0} \subset S_{0}^{*}$. Let $\mathbb{T}=T_{0}^{* *}$ and $\mathbb{S}=S_{0}^{* *}$. Then $\mathbb{T} \subset \mathbb{S}^{*}$ and $\mathbb{S} \subset \mathbb{T}^{*}$. 
We next check that $\mathscr{D}_{00}$, respectively $\mathscr{D}_{00}^{*}$, are cores of $\mathbb{T}$, respectively $\mathbb{S}:$ With $f \in \mathscr{H}$, let the $\left\{f_{n}\right\} \subset \mathscr{H}_{0}$ be a sequence such that $\left\|f_{n}-f\right\|_{2} \underset{n=\infty}{\longrightarrow} 0$, we have, for all $b_{0} \in \mathscr{D}^{\prime}$

$$
\left\{\begin{array}{l}
\pi\left(b_{0}^{\hat{0}}\right) f_{n} \underset{n=\infty}{\longrightarrow} \pi\left(b_{0}^{\hat{0}}\right) f \\
\text { respectively } \pi\left(b_{0}^{\hat{\imath}}\right)^{*} f_{n} \underset{n=\infty}{\longrightarrow} \pi\left(b_{0}\right)^{*} f
\end{array}\right.
$$

whilst

$$
\left\{\begin{array}{l}
\mathbb{T} \pi\left(b_{0}^{\wedge}\right) f_{n}=\pi\left\{\left(T^{\prime} b_{0}\right)^{\wedge}\right\} f_{n} \underset{n=\infty}{\longrightarrow} \pi\left\{\left(T^{\prime} b_{0}\right)^{\wedge}\right\} f=\mathbb{T} \pi\left(b_{0}^{\wedge}\right) f \\
\text { respectively } \mathbb{S} \pi\left(\hat{b_{0}}\right)^{*} f_{n}=\pi\left\{\left(T^{\prime} b_{0}\right)^{\wedge}\right\}^{*} f_{n} \underset{n=\infty}{\longrightarrow} \pi\left\{\left(T^{\prime} b_{0}\right)^{\wedge}\right\}^{*} f=\mathbb{S} \pi\left(b_{0}\right)^{*} f .
\end{array}\right.
$$

Properties (44) and (45) are easily checked from the definitions (42), (43) taking account of Lemma 3. Let $f \in \mathscr{D}_{00}$, we have $f=\pi\left(b_{0}\right) f_{0}$ with $f_{0} \in \mathscr{H}_{0}$, thus, by Lemma $3, i\left(f=\hat{\pi}\left(\hat{b_{0}} a_{0}\right), a_{0} \in \mathscr{A}\right.$ such that $\hat{\pi}\left(a_{0}\right)=i\left(f_{0}\right)$, whereby $a=\hat{b_{0}} a_{0} \in \mathscr{D}$ by (iii') in Lemma 4. Now, by (42) and (26).

$i(\mathbb{I} f)=i\left\{\pi\left[\left(T^{\prime} b_{0}\right)^{\wedge}\right] f_{0}\right\}=\hat{\pi}\left(\left(T^{\prime} b_{0}\right)^{\wedge} a_{0}\right)=\hat{\pi}\left(T\left(b_{0} a_{0}\right)\right)=\hat{\pi}(T a)$.

On the other hand let $h \in \mathscr{D}_{00}^{*}$, we have $h=\pi\left(\bar{b}_{0}\right) f_{0}$, thus by (43) and (27)

$$
\begin{aligned}
\lambda\left(\mathbb{T}^{*} h\right) & =\lambda\left(\pi \overline{\left(T^{\prime} b_{0}\right)} f\right)=\pi \overline{\left(T^{\prime} b_{0}\right)} \lambda(f) \\
& =\pi\left(\bar{T}^{\prime} \bar{b}_{0}\right) \lambda(f) .
\end{aligned}
$$

Now (44) and $\left(T_{1}\right),\left(T_{2}\right)$ readily imply that $\mathscr{D}_{00}$ is right and left $G$-invariant and that $\mathbb{T}$ commutes with $\lambda_{s}$ and $\lambda_{s}^{\prime}$, then $\mathbb{T}$ is affiliated with the center $\mathscr{Z}$ of $\mathscr{R}$. Hence $\mathbb{S}=\mathbb{T}^{*}$.

We now proceed to prove (46). Since two closed operators affiliated to an abelian von Neumann algebra, one of which extends the other, must coincide, it suffices to (46) with the equality sign replaced by $C$. For this in turn it suffices to show that

$$
W(\mathbb{T} f \otimes \mathbb{T} g)=(\mathbb{T} \otimes \mathbb{1}) W(f \otimes g), \quad f \in \mathscr{D}_{00}, \quad g \in \mathscr{D}_{\mathbb{T}},
$$

or that both sides of (54) have the same scalar product with $h \otimes k, h \in \mathscr{D}_{00}^{*}, k \in \mathscr{H}$. Taking account of (A3) in the appendix, this comes to

$$
(\mathbb{T} g \mid \lambda(\overline{\mathbb{T}} f \cdot h) k)=\left(g \mid \lambda\left(\bar{f} \cdot \mathbb{T}^{*} h\right) k\right), \quad \begin{cases}f \in \mathscr{D}_{00}, & g \in \mathscr{D}_{\mathbb{T}} \\ h \in \mathscr{D}_{00}^{*}, & k \in \mathscr{H} .\end{cases}
$$

We shall need to know that

$$
\lambda(\varphi \cdot \pi(b) \xi)=\pi\left(\pi_{*}(\varphi) \cdot\left(n \pi_{*}(\xi)\right)\right), \quad \varphi \in A(G), \quad b \in \mathscr{B}, \quad \xi \in H_{0},
$$

indeed, for $\psi \in A(G)$,

$$
\begin{aligned}
\langle\lambda(\varphi \cdot \pi(b) \xi), \psi)\rangle & =\langle\varphi \cdot \lambda(\pi(b) \xi), \psi\rangle \quad \text { by Lemma A6 } \\
& =\langle\lambda(\pi(b) \xi), \varphi \psi\rangle=\langle\pi(b) \lambda(\xi), \varphi \psi\rangle \quad \text { by }(27) \\
& =\left\langle\pi\left(b \hat{\pi}_{*}(\xi)\right), \varphi \psi\right\rangle=\left\langle b \hat{\pi}_{*}(\xi), \pi_{*}(\varphi \psi)\right\rangle \\
& =\left\langle b \hat{\pi}_{*}(\xi), \pi_{*}(\varphi) \pi_{*}(\psi)\right\rangle=\left\langle\pi_{*}(\varphi) \cdot\left(b \pi_{*}(\xi)\right), \pi_{*}(\psi)\right\rangle \\
& =\left\langle\pi\left(\pi_{*}(\varphi) \cdot b \pi_{*}(\xi)\right), \psi\right\rangle .
\end{aligned}
$$


Now on the one hand, with $a \in \mathscr{A}$, such that $\hat{\pi}(a)=i(f)$ and $b \in \overline{\mathscr{D}}^{\prime}, \xi \in \mathscr{H}_{0}$ such that $\pi(b) \xi=h$, we have

$$
\begin{aligned}
\lambda(\overline{\mathrm{T} f} \cdot h) & =\lambda(\overline{\mathrm{T} f} \cdot(\pi(b) \xi)) \\
& =\pi\left(\pi_{*}(\overline{\mathrm{T} f}) \cdot\left(b \hat{\pi}_{*}(\xi)\right)\right) \quad \text { by }(56) \\
& =\overline{\pi\left((T a) \cdot\left(\bar{b} \hat{\pi}_{*}(\xi)\right)\right)} .
\end{aligned}
$$

And by (44), (45), $(T a) \cdot \overline{b \hat{\pi}_{*}(\xi)} \in(T \mathscr{D}) \cdot \mathscr{D}^{\prime} \subset \mathscr{D}^{\prime} \quad[\mathrm{cf}$. Lemma 4 (iv)], thus $\lambda(\mathbb{T} f$ . h) $k \in \mathscr{D}_{0}^{*} \subset \mathscr{D}_{\mathbb{T}}$ * and

$$
\begin{aligned}
\mathbb{T}^{*} \lambda(\overline{\mathbb{T} f} \cdot h) k & =\overline{\pi\left(T^{\prime}\left(T a \cdot \overline{b \hat{\pi}_{*}(\xi)}\right)\right.} k \\
& =\pi\left(\bar{a} \cdot \bar{T}^{\prime} b \hat{\pi}_{*}(\xi)\right) k .
\end{aligned}
$$

On the other hand, we have, by (56) and (45),

$$
\begin{aligned}
\lambda\left(\bar{f} \cdot \mathbb{T}^{*} h\right) k & =\bar{f} \cdot \lambda\left(\mathbb{T}^{*} \pi(b) \xi\right) k \\
& =\bar{f} \pi\left(\bar{T}^{\prime} b \hat{\pi}_{*}(\xi)\right) k=\pi\left(\hat{\pi}(\bar{f}) \cdot\left(\bar{T}^{\prime} b \hat{\pi}_{*}(\xi)\right) k\right. \\
& =\pi\left(\bar{a} \cdot\left(\bar{T}^{\prime} b \hat{\pi}_{*}(\xi)\right) k ;\right.
\end{aligned}
$$

we proved (55), i.e., (46).

From this and Theorem A5 in the appendix now follows

Theorem 6. Let $G$ be a locally compact group, with $\mathscr{A}$ and $\mathscr{B}$ a pair of Banach algebras in duality satisfying assumptions I through $V$ above (for example one of the pairs in (7)). Let $T$ be a linear $\sigma(\mathscr{A}, \mathscr{B})$-closed operator on $\mathscr{A}$ with $\sigma(\mathscr{A}, \mathscr{B})$-dense domain $\mathscr{D}$ fulfilling properties $\left(T_{1}\right),\left(T_{1}^{\prime}\right)$, and $\left(T_{2}\right)$ above. Then there exists an element $s_{0}$ in the center $\mathscr{Z}(G)$ of $G$ and a continuous one-parameter subgroup $\{g(t)\}$ of $\mathscr{Z}(G)$ such that for every $a \in \mathscr{D}$ and $s \in G$ there is a $u \in H^{\infty}(D), D=\{z \in \mathbb{C} ;-1 \leqq \operatorname{Im} z \leqq 0\}$, such that

$$
\left\{\begin{array}{l}
u(t)=\hat{\pi}(a)\left(g(t)^{-1} s\right) \\
u(t-i)=\hat{\pi}(T a)\left(s_{0} g(t)^{-1} s\right), \quad t \in \mathbb{R} .
\end{array}\right.
$$

Conversely with $G, \mathscr{A}$ and $\mathscr{B}$ as above, $s_{0} \in G$, and $\{g(t)\}$ a continuous oneparameter subgroup of $G$, there is a unique densely defined closed operator $T$ on $\mathscr{A}$ such that for every a in its domain and $s \in G$ we have a $u \in H^{\infty}(D)$ giving rise to (60).

For the proof we need the three following lemmas.

Lemma 7. With the assumptions of Theorem 6, let

$$
\left\{\begin{array}{l}
\mathscr{D}_{A}=\left\{\varphi \in A ; \pi_{*}(\varphi) \in \mathscr{D} \text { and } T \pi_{*}(\varphi) \in \pi_{*}(A)\right\} \\
\mathscr{L}_{L^{1}}^{\prime}=\left\{f \in L^{1} ; \hat{\pi}_{*}(f) \in \mathscr{D}^{\prime} \text { and } T \hat{\pi}_{*}(f) \in \hat{\pi}_{*}\left(L^{1}\right)\right\} .
\end{array}\right.
$$

Then $\mathscr{D}_{A}$ is a dense subalgebra of $A$ and $\mathscr{D}_{L^{1}}^{\prime}$ is a dense ideal of $L^{1}$. Furthermore the linear operators $T_{A}$ on $A$ and $T_{L^{1}}^{\prime}$ on $L^{1}$ defined by the following commutative diagrams are both closed

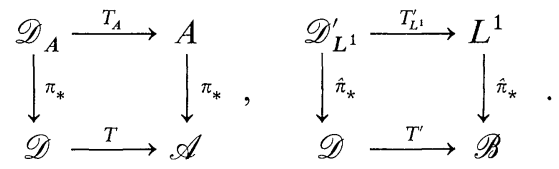


The closed operator $T_{L^{\infty}}$ on $L^{\infty}$ defined as the adjoint of $T_{L^{1}}^{\prime}$ then gives rise to the following commutative diagram

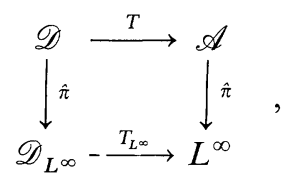

the domain $\mathscr{D}_{L^{\infty}}$ of $T_{L^{\infty}}$ containing $\hat{\pi}(\mathscr{D})$ as a core.

Remark 1. It follows that $T_{L^{1}}^{\prime}$ is the adjoint of $T_{L^{\infty}}$ and that $T_{L^{\infty}}$ fulfills the conditions $\left(T_{1}\right),\left(T_{1}^{\prime}\right)$, and $\left(T_{2}\right)$ with $\mathscr{A}=\mathrm{L}^{\infty}$ and $\mathscr{B}=L^{1}$. The conclusions of Lemmas 4 and 5 thus hold for $T=T_{L^{\infty}}, T^{\prime}=T_{L^{1}}^{\prime}, \mathscr{A}=L^{\infty}$ and $\mathscr{B}=L^{1}$.

Remark 2. Diagram (63) shows that the direct statement in Theorem 6 holds for the dual pair $(\mathscr{A}, \mathscr{B})$ and the operator $T$ if it holds for the dual pair $\left(L^{\infty}, L^{1}\right)$ and the operator $T_{L^{\infty}}$ : indeed for any $a \in \mathscr{D},(\hat{\pi}(a), \hat{\pi}(T a))$ belongs to the graph of $T_{L^{\infty}}$.

Proof of Lemma 7. Since Graph $T_{A}=\left(\pi_{*} \times \pi_{*}\right)^{-1}$ Graph $T$ and Graph $T_{L^{1}}^{\prime}$ $=\left(\hat{\pi}_{*} \times \hat{\pi}_{*}\right)^{-1}$ Graph $T^{\prime}$, closedness of $T_{A}$ and $T_{L^{1}}^{\prime}$ follow from that of $T$ and $T^{\prime}$.

Now we have the inclusions

$$
\begin{gathered}
\mathscr{D}_{A} \supset .\left(\hat{\pi} \circ \pi_{*}\right)^{-1}\left\{i\left(\mathscr{D}_{00}\right)\right\}, \\
\mathscr{D}_{L^{1}}^{\prime} \supset \mathrm{i}\left(\mathbb{T} \mathscr{D}_{00}\right) \cdot \mathrm{i}\left(\overline{\mathscr{D}}_{00}^{*}\right.
\end{gathered}
$$

from which the density of $\mathscr{D}_{A}$ and $\mathscr{D}_{L^{1}}^{\prime}$ follow: indeed the preimage of $i\left(\mathscr{D}_{00}\right)$ for $\hat{\pi} \circ \pi_{*}$ is evidently dense in $A$. And $i\left(\mathscr{D}_{00}^{*}\right) \cdot i\left(\mathbb{T} \mathscr{D}_{00}\right)$ is dense in $L^{1}$ since $\overline{\mathscr{D}}_{00}^{*}$ and $\mathbb{T} \mathscr{D}_{00}$ are dense in $\mathscr{H}$ (the latter since $\mathscr{D}_{00}$ is a core of the nonsingular operator $\mathbb{T}$ ).

We prove (64): let $f \in \mathscr{D}_{00}, a \in \mathscr{A}$ and $\varphi \in A$ with $\hat{\pi}(a)=i(f)$ and $\pi_{*}(\varphi)=a$. By Lemma $5 \hat{\pi}(T a)=i(\mathbb{T} f)$, thus $\hat{\pi}(T a)$ belongs to $\left(\hat{\pi} * \pi_{*}\right)(A)$, thus $T a$ belongs to $\pi_{*}(A)$.

We prove (65): We first claim that

$$
\begin{array}{cl}
\hat{\pi}_{*}(i(\varphi) \cdot(\pi(b) \xi))=\pi_{*}(\varphi) \cdot\left(b \hat{\pi}_{*}(\xi)\right), & \varphi \in A(G) \cap L^{2}(G), \quad b \in \mathscr{B}, \\
& \xi \in L^{1}(G) \cap L^{2}(G) .
\end{array}
$$

In fact, if $\left\{f_{k}\right\}$ is a net in $L^{1}(G)$ such that $\left\{\hat{\pi}_{*}\left(f_{k}\right)\right\}$ converges to $b$ in the $\sigma(\mathscr{B}, \mathscr{A})$ topology, then $\lambda\left(f_{k}\right)$ converges to $\pi(b) \sigma$-weakly, so we have, for $a \in \mathscr{A}$,

$$
\begin{aligned}
\left\langle a, \pi_{*}(\varphi) \cdot\left(b \hat{\pi}_{*}(\xi)\right)\right\rangle & =\lim _{k}\left\langle a, \pi_{*}(\varphi) \cdot\left(\hat{\pi}_{*}\left(f_{k}\right) \hat{\pi}_{*}(\xi)\right)\right\rangle \\
& =\lim \left\langle\pi_{*}(\varphi) a, \hat{\pi}_{*}\left(f_{k} \xi \xi\right)\right\rangle=\lim \left\langle\hat{\pi}(a) i(\varphi), \lambda\left(f_{k}\right) \xi\right\rangle \\
& \left.\left.=\lim \overline{(\hat{\pi}(a) i(\varphi)} \mid \lambda\left(f_{k}\right) \xi\right)=\overline{(\hat{\pi}(a) i(\varphi)} \mid \pi(b) \xi\right) \\
& =\langle\hat{\pi}(a), i(\varphi) \cdot(\pi(b) \xi)\rangle .
\end{aligned}
$$

Hence (66) follows. Let $h=i(\mathbb{T} f) \cdot(\pi(b) \xi)$ with $f \in \mathscr{D}_{00}, b \in \mathscr{D}^{\prime}$ and $\xi \in \mathscr{H}_{0}$. Since $\mathbb{T} f \in L^{2}(G) \cap A(G)$, we have, with $a \in \mathscr{A}$ such that $\pi(a)=i(f)$,

$$
\hat{\pi}_{*}(h)=\hat{\pi}_{*}(\hat{\pi}(T a) \cdot \pi(b) \xi)=T a \cdot(b \hat{\pi}(\xi)) .
$$


Thus $\hat{\pi}_{*}(h) \in(T \mathscr{D}) \cdot \mathscr{D}^{\prime} \subset \mathscr{D}^{\prime}$ (cf. Lemma 4); and

$$
\left.T^{\prime} \hat{\pi}_{*}(h)=a \cdot\left[\left(T^{\prime} b\right) \hat{\pi}_{*}(\xi)\right)\right]=\hat{\pi}_{*}\left(i(f) \cdot\left(\pi\left(T^{\prime} b\right) \xi\right)\right) .
$$

Lemma 8. $\mathscr{D}_{L^{1}}^{\prime}$ contains a bounded approximate identity of $L^{1}$.

Proof. For each relatively compact neighborhood $U$ of the unit $e \in G$, set

$$
e_{U}=\chi_{U} / \int \chi_{U}(s) d s
$$

$\left(\chi_{U}\right.$ the characteristic function of $U$ ): we thus get an approximate identity of $\left\{e_{U}\right\}$ of $L^{1}$. Now, by the density of $\mathscr{D}_{L^{1}}^{\prime}$ in $L^{1}$, there are elements $f_{U} \in \mathscr{D}_{L^{1}}^{\prime}$ such that

$$
\left\|f_{U}-e_{U}\right\|_{1} \leqq \int \chi_{U}(s) d x \text { and }\left\|f_{U}\right\|_{1} \leqq 1 .
$$

Obviously $\left\{f_{U}\right\}$ is a bounded approximate identity of $L^{1}$.

Lemma 9. $i\left(\mathscr{L}_{00}\right)$ is a core of $T_{L^{\infty}}$.

Proof. Applying Lemma 4 (i), (iii') to $T_{L^{\infty}}$ and $T_{L^{1}}^{\prime}$ we have that, for $f \in \mathscr{D}_{L^{1}}^{\prime \prime}$ and $u \in \mathscr{D}_{L^{\infty}}, f^{\wedge} u \in \mathscr{D}_{L^{\infty}}$ and

$$
T_{L^{\infty}}\left(f^{\wedge} u\right)=f^{\wedge}\left(T_{L^{\infty}} u\right)=\left(T_{L^{1}}^{\prime} f\right)^{\wedge} u .
$$

With $\left\{f_{U}\right\}$ the approximate identity described in Lemma $8, u \in \mathscr{D}_{L^{\infty}}$ and $g \in L^{1}$, we have

$$
\left\langle f_{U}^{\wedge} u, g\right\rangle=\left\langle u, f_{U} g\right\rangle \underset{U}{\longrightarrow}\langle u, g\rangle
$$

whilst, from (70)

$$
\left\langle T_{L^{\infty}}\left(f_{U^{n}}^{\wedge} u, g\right\rangle=\left\langle f_{U}^{\wedge}\left(T_{L^{\infty}} u\right), a\right\rangle=\left\langle T_{L^{\infty}} u, f_{U} g\right\rangle \underset{U}{\longrightarrow}\left\langle T_{L^{\infty}} u, g\right\rangle .\right.
$$

Thus $\mathscr{D}_{L^{1}}^{\prime} \mathscr{D}_{L^{\infty}}$ is a core of $\mathscr{D}_{L^{\infty}}$. Now let $f \in \mathscr{D}_{L^{1}}^{\prime}$ and $u \in \mathscr{D}_{L^{\infty}}$. Since $i\left(\mathscr{H}_{0}\right)$ is $\sigma\left(L^{\infty}, L^{1}\right)$-dense in $L^{\infty}$ there is a sequence $\left\{\xi_{j}\right\} \subset \mathscr{H}_{0}$ with $i\left\{\xi_{j}\right\} \underset{j=\infty}{\longrightarrow} u$. We have then, for all $g \in L^{1}$

$$
\left\langle f^{\wedge} i\left(\xi_{j}\right), g\right\rangle=\left\langle i\left(\xi_{j}\right), f g\right\rangle \underset{j=\infty}{\longrightarrow}\langle u, f g\rangle=\left\langle f^{\wedge} u, g\right\rangle
$$

whilst, from (70)

$$
\begin{aligned}
\left\langle T_{L^{\infty}}\left\{f^{\wedge} i\left(\xi_{j}\right)\right\}, g\right\rangle & =\left\langle i\left(\xi_{j}\right),\left(T_{L^{1}}^{\prime} f\right) g\right\rangle=\left\langle\left(T_{L^{1}}^{\prime} f\right)^{\wedge} i\left(\xi_{j}\right), g\right\rangle \\
& \cdot \underset{i=\infty}{\longrightarrow}\left\langle u,\left(T_{L^{1}}^{\prime} f\right) g\right\rangle=\left\langle\left(T_{L^{1}}^{\prime} f\right)^{\wedge} u, g\right\rangle=\left\langle T_{L^{\infty}}\left(f^{\wedge} u\right), g\right\rangle .
\end{aligned}
$$

We proved that $\mathscr{D}_{L^{1}}^{\prime \prime} i\left(\mathscr{H}_{0}\right)=i\left(\mathscr{D}_{00}\right)$ is a core of $T_{L^{\infty}}$.

Proof of Theorem 6. By the Duality Theorem (Theorem A5 in the appendix) there exists an $s_{0} \in G$ and a one-parameter subgroup $\{g(t)\}$ of $G$ such that

$$
\mathbb{U}=\lambda_{s_{0}} ; \quad|\mathbb{T}|^{i t}=\lambda_{g(t)}, \quad t \in \mathbb{R},
$$

where $\mathbb{T}=\mathbb{U}|\mathbb{T}|$ is the polar decomposition of $\mathbb{T}$. Furthermore, since $\mathbb{T}$ is affiliated with the center of $\mathscr{R}, s_{0}$ and $\{g(t)\}$ are both central. The domain $\mathscr{D}_{\mathbb{T}}$ of $\mathbb{T}$ then 
consists for all those $f \in H$ such that the $H$-valued continuous function

$$
t \in \mathbb{R} \rightarrow \lambda_{g(t)} f=|\mathbb{T}|^{i t} f \in \mathscr{H}
$$

admits an extension to a bounded continuous function $z \in S \rightarrow f(z) \in \mathscr{H}$ on the strip

$$
S=\{z \in \mathbb{C} ;-1 \leqq \operatorname{Im} z \leqq 0\}
$$

which is holomorphic in the interior of $S$. We then have

$$
f(t-i)=\lambda_{g(t)}|\mathbb{T}| f, \quad t \in \mathbb{R} .
$$

Let now $f \in \mathscr{D}_{00}$. We noted that Lemma 5 applies to the case $\mathscr{A}=L^{\infty}, \mathscr{B}=L^{1}$, $T=T_{L^{\infty}}:(44)$ then yields that $i(f) \in \mathscr{D}_{L^{\infty}}$ with

$$
T_{L^{\infty}} i(f)=i(\Pi f) .
$$

Thus if we set

$$
F_{f, s}(z)=i(f(z))(s), \quad s \in G, \quad z \in S,
$$

we have, by (18), using the fact that $i \circ \lambda_{g}=\lambda_{g} \circ i, g \in G$, that

$$
F_{f, s}(t)=i\left(\lambda_{g(t)} f\right)(s)=i(f)\left(g(t)^{-1} s\right), \quad s \in G, \quad t \in \mathbb{R},
$$

whilst

$$
\begin{aligned}
F_{f, s}(t-i) & =i\left(\lambda_{g(t)}|\mathbb{T}| f\right)(s)=i\left(\lambda_{g(t)} \lambda_{s_{0}-1} \mathbb{T} f\right)(s) \\
& =i(\mathbb{T} f)\left(s_{0} g(t)^{-1} s\right) \quad s \in G, \quad t \in \mathbb{R} . \\
& =T_{L^{\infty}} i(f)\left(s_{0} g(t)^{-1} s\right) .
\end{aligned}
$$

We have thus established property (60) for $T=T_{L^{\infty}}$ and the elements of $\mathscr{A}=L^{\infty}$ belonging to $i\left(\mathscr{D}_{00}\right)$. In order to extend this property to all elements of $\mathscr{D}_{L^{\infty}}$ we now use the fact that $\left\{\lambda_{g(t)}\right\}$ acting on $L^{\infty}$ is a bounded continuous one-parameter group and this gives rise to the analytic generator in the sense of Ciorănescu and Zsidó [11], a $\sigma\left(L^{\infty}, L^{1}\right)$-closed operator $U(-i)$ with domain consisting in all $f \in L^{\infty}$ such that $t \rightarrow \lambda_{g(t)} f$ admits an $L^{\infty}$-valued bounded holomorphic function $F(z)$ on $S$, with

$$
U(-i) f=F(-i) .
$$

The above discussion shows that

$$
T_{L^{\infty}} i(f)=\lambda_{s_{0}} U(-i) i(f), \quad f \in \mathscr{D}_{00} .
$$

Since $i\left(\mathscr{D}_{00}\right)$ is a core of $T_{L^{\infty}}, \lambda_{s_{0}} U(-i)$ is an extension of $T_{L^{\infty}}$. Therefore, if $g \in \mathscr{D}_{L^{\infty}}$, there exists an $L^{\infty}$-valued bounded function $\mathrm{F}$ on $S, \sigma\left(L^{\infty}, L^{1}\right)$-continuous on $S$ and holomorphic in its interior, such that

$$
F(t)=\lambda_{g(t)} f ; \quad F(t-i)=\lambda_{g(t)} \lambda_{s_{0}}^{-1} T f, \quad t \in \mathbb{R} .
$$

Let $\Phi$ be a left translation invariant lifting of $L^{\infty}$ to $\mathscr{L}^{\infty}[13] .{ }^{6}$ We then set

$$
F_{s}(z)=\Phi(F(z))(s), \quad s \in G, \quad z \in S .
$$

$6 \mathscr{L}^{\infty}$ denotes the algebra of bounded measurable functions (defined everywhere without identification modulo null sets) 
Since $\Phi$ is a norm-continuous isomorphism of $L^{\infty}$ into the algebra of bounded measurable functions on $G, F_{s}$ is holomorphic inside $S$, and we have

$$
\left\{\begin{array}{l}
F_{s}(t)=f\left(g(t)^{-1} s\right) \\
F_{s}(t-i)=\left(T_{L^{\infty}} f\right)\left(s_{0} g(t)^{-1} s\right) .
\end{array}\right.
$$

We ended the proof of the direct statement in Theorem 6 (cf. Remark 2 above).

The converse part of Theorem 6 is obtained by taking $T=\lambda_{s_{0}} U(-i), U(-i)$ the analytic generator of the automorphism group $t \rightarrow \lambda_{g(t)}$ of $\mathscr{A}$ [11].

\section{Density of the Two-Point Functions of Invariant States}

Our two last sections are minor modifications of the corresponding sections of [1], of which we adopt exactly the notation and definitions, $G$ being now a (noncommutative) locally compact group.

For the discussion preceding Propositions 3.4 and 3.5 of [1] to go through in the noncommutative group case we have merely to replace the assumption $\operatorname{Sp} U=\hat{G}$ by the following corresponding requirement :

(R) The unitary representation $U$ contains weakly the regular representation $\lambda$ of $G$.

The above-mentioned propositions now generalize to the following density results for the two-point functions of invariant states:

Theorem 10. Let $\omega$, with the notation of $[1,3]$ be an invariant state of the $C^{*}$-system $\{\mathfrak{U}, G, \alpha\}$. We have that

(i) $\mathscr{F}$ (or $\mathscr{G}$, or $\mathfrak{f}$, or $\mathfrak{g}$ ) is weak $*$ total in $B$ iff (R) holds.

(ii) these sets are even weak $*$ dense in $B$ if we assume in addition the existence of an action $\tau$ of a locally compact group $H$ on $\mathfrak{A}$ commuting with $\alpha$ and such that $\omega$ is $\tau$-invariant and $\tau$-clustering.

Theorem 11. Let again $\omega$ be an $\alpha$-invariant state of the $C^{*}$-system $\{\mathfrak{U}, G, \alpha\}$ with the notation of $[1,3]$. And assume the existence of an action $\tau$ of an amenable group $H$ on $\mathfrak{A}$, commuting with $\alpha, \omega$ asymptotically abelian, and for which $\omega$ is weakly $\tau$ clustering. If $(\mathrm{R})$ holds the sets $\mathscr{F}, \mathscr{G}, \mathfrak{f}$, and $\mathfrak{g}$ are all weak $*$ total in $L^{\infty}$. These sets are even weak * dense in $L^{\infty}$ if $\omega$ is $\tau$-clustering.

\section{Criteria for the KMS Property}

The argument of [1, Sect. 4] are valid for the following generalization, to the case of a non-abelian $G$, of Theorems 4.1 and 4.3 in [1].

Theorem 12. Let $\omega$ be an $\alpha$-invariant state of the $C^{*}$-system $\{\mathfrak{U}, G, \alpha\}$ with the notation of $[1,3]$ and assume that $(\mathrm{R})$ holds. Assume further that $\omega$ is extremal $\tau$ invariant for an action $\tau$ of an amenable group $H$ on $\mathfrak{A}$ which is $\omega$-asymptotically abelian and commutes with $\alpha$. Let $(\mathscr{A}, \mathscr{B})$ be one of the pairs $\left(B, C^{*}\right)$ or $\left(L^{\infty}, L^{1}\right)$. If there is a closable linear operator $T_{0}$ in $\mathscr{A}$, with domain the linear span of $\mathscr{F}(\mathfrak{f})$, such that $T_{0} F_{A B}=G_{A B}\left(T_{0} f_{A}=g_{A B}\right)$ for all $A, B \in \mathfrak{A}$, then $\omega$ is KMS for some continuous one-parameter subgroup of the center of $G$ - and conversely. 
These conclusions are maintained under the following alterings of assumptions

(i) $\tau$ is assumed $\tau$-invariant and $\tau$-clustering (instead of merely extremal $\tau$-invariant).

(ii) $T_{0}$ is merely defined on $\mathscr{F}(\mathfrak{f})$ and closable in $\mathscr{A}$ (the linearity assumption for $T_{0}$ and its domain is suppressed).

The fact that the KMS condition implies in turn the existence of the operator $T_{0}$ follows by taking, for the letter, the restriction to $\mathscr{F}(\mathfrak{f})$ of the analytic generator of Ciorănescu and Zsidó [11].

We conclude with a comment on the physical context of this paper. Our general aim is a description, within the field-theoretic frame proposed by Rudolf Haag, of the general features of thermodynamical (i.e. finite temperature) equilibrium states. Haag's scheme for local physics consists in the representation theory of a $C^{*}$-algebra endowed with a relativistic local and automorphic structure [an inductive limit of local algebras with the natural inclusion and (space-like) commutativity properties, acted upon by the Lorentz group equivariantly with respect to the local structure]. In this scheme equilibrium states are defined as the extremal invariant states for the one-parameter groups of time-like translations. The first main challenge of the theory is then to give a basic explanation for the occurrence of the KMS condition universally observed in this context (the so called Gibbs structure - the positivity of the energy in the vacuum representation is a limiting case). Since the Gibbs structure is shared by relativistic and nonrelativistic situations, we expect an explanation based on the automorphic rather than the local structure - in fact on these features of the automorphic structure common to relativistic and non-relativistic situations. This actually happens for the two kinds of explanations proposed thus far, based on the postulated dynamical stability, resp. the passiveness, of the equilibrium states [4, $14]^{7}$. Now these derivations relate the hitherto mysterious KMS condition to physically necessary properties of the equilibrium states, and thus provide a physical understanding of the Gibbs structure. However the problem remains to explain the latter from basic properties of the interaction. It thus seems worthwhile to try and reformulate the KMS property in a way hopefully more closely related to these basic properties. In this connection we pursue the project of deriving the Gibbs structure from a combination of locality (here technically asymptotic abelianness) and a modernized form, stated within the algebraic quantum context, of Boltzmann's ergodicity (pervading nature of the interaction). The latter property is clearly a general property of physical interactions, hitherto insufficiently exploited, and which could tentatively be expressed as an "insplitability" of the dynamical $C^{*}$-system - or the presumably related fact that an appropriate subgroup of the Lorentz group already contains the automorphisms with which it commutes (few constants of the motion). We hope that the result presented in this paper relates to such a program, since we believe that the

7 Passiveness is a condition related to the second principle of thermodynamics. We do not mention here the explanations based on extremum principles - confined to models of the (hopefully provisional) absence of the entropy concept in the algebraic frame. We recall that the fact that KMS is for a mixture of time and gauge on the field algebra is well understood algebraically $[3,15]$ 
existence of the map $T: F_{A B} \rightarrow G_{A B}$ is connected with ergodicity (as a tightness condition - this however still has to be demonstrated). The present extension of an earlier result to the non-commutative group case is motivated in this context by the possibility that ergodicity should only hold with respect to non-abelian subgroups of the Lorentz group (possibly the whole Lorentz group).

\section{Appendix. Duality of Locally Compact Groups}

This appendix, added for self-containment, describes the duality of locally compact groups (Tannaka, Stinespring, Eymard, Tasuuma, Saito) in an operatortheoretic spirit.

Theorem A1. Let $G$ be a locally compact group, let $\mathscr{H}^{\otimes 2}=L^{2}(G) \otimes L^{2}(G)$ $=L^{2}(G \times G)$, and let $W: \mathscr{H}^{\otimes 2} \rightarrow \mathscr{H}^{\otimes 2}$ be the operator

$$
(W f)(s, t)=f(s, s t), \quad f \in \mathscr{H}^{\otimes 2}, \quad s, t \in G .
$$

Let furthermore $\mathscr{G}$ be the set of nonvanishing bounded operators on $\mathscr{H}=L^{2}(G)$ fulfilling

$$
W(T \otimes T)=(T \otimes \mathbb{1}) W .
$$

We have $\mathscr{G}=\left\{\lambda_{g} ; g \in G\right\}$ the set of representatives of $G$ in its left regular representation.

We prove this theorem by establishing a succession of lemmas.

Lemma A2. $\lambda_{s} \in \mathscr{G}$ for all $s \in G$. Consequently $\lambda$ is quasiequivalent to $\lambda \otimes \lambda$, and $A(G)$ is $a *$-subalgebra of $B(G)$.

Proof. For $f \in \mathscr{H}^{\otimes 2}$ one has $\left(\lambda_{u} \otimes \lambda_{u}\right) f(s, t)=f\left(u^{-1} s, u^{-1} t\right)$ thus $W(T \otimes T) f(s, t)$ $=f\left(u^{-1} s, u^{-1} s t\right)=(T \otimes \mathbb{1}) W f(s, t)$. Since $\mathscr{R}(G)$ is a standard von Neumann algebra, each of its normal forms is a vector form, thus $\lambda \otimes \lambda \sim \lambda$ implies that the set $A(G)$ of normal forms is multiplicative.

Our next lemma states properties of the operator $W$.

Lemma A3. The operator $W$ defined in (A1) is unitary; belongs to the von Neumann tensor product $m\left(L^{\infty}(G)\right) \otimes \mathscr{R}(G)$ (where $m$ is the representation of $L^{\infty}(G)$ by pointwise multiplication on $\mathscr{H}$ ); commutes with $C$ (where $C f=\bar{f}$ ); and fulfills the property

$$
\begin{aligned}
(W(f \otimes g) \mid h \otimes k) & =\left\langle g * k^{\nu}, \bar{f} \cdot h\right\rangle, \quad f, g, h, k \in \mathscr{H} \\
& =(g \mid \lambda(\bar{f} \cdot h\rangle k),
\end{aligned}
$$

where $g * k^{2} \in A(G)$ and $\bar{f} \cdot h \in L^{1}(G)$.

Proof. One has obviously $W^{*}=W^{-1}$ with

$$
W^{-1} f(s, t)=f\left(s, s^{-1} t\right), \quad s, t \in G .
$$


We recall that the commutant $\mathscr{R}^{\prime}(G)$ of $\mathscr{R}(G)$ is generated by the $\lambda_{s}^{\prime}, s \in G$, where ${ }^{8}$

$$
\left(\lambda_{r}^{\prime} f\right)(s)=\Delta(r)^{1 / 2} f(s r), \quad r, s \in G .
$$

Now $W$ commutes with $\mathbb{1} \otimes \lambda_{s}^{\prime}, s \in G$ :

$$
\begin{aligned}
W\left(\mathbb{1} \otimes \lambda_{r}^{\prime}\right) f(s, t) & =\Delta(r)^{1 / 2} f(s, s t r), \quad f \in \mathscr{H}, \quad r, s, t \in G \\
& =\left(\mathbb{1} \otimes \lambda_{r}^{\prime}\right) W f(s, t) .
\end{aligned}
$$

Our claim that $W$ belongs to $m\left(L^{\infty}(G)\right) \bar{\otimes} \mathscr{R}(G)$ then follows via maximal abelianness of $L^{\infty}(G)$ from the fact that $W$ commutes with all $m_{g} \otimes \mathbb{1}, g \in L^{\infty}(G)$, where

$$
m_{g} \xi(s)=g(s) f(s), \quad g \in L^{\infty}(G), \quad \xi \in \mathscr{H}, \quad s \in G .
$$

Indeed

$$
\left(m_{g} \otimes \mathbb{1}\right) W f(s, t)=g(s) f(s, s t)=W\left(m_{g} \otimes \mathbb{1}\right) f(s, t) .
$$

$W$ obviously commutes with $C$. On the other hand

$$
\begin{aligned}
(W(f \otimes g) \mid h \otimes k) & =\int \bar{f}(s) \bar{g}(s t) h(s) k(t) d s d t \\
& =\int\left(\bar{f} h\left(\bar{g} * k^{y}\right)\right)(s) d x,
\end{aligned}
$$

(A3) thus follows from (11).

The next lemma shows that $\mathscr{G}$ consists of unitaries of $\mathscr{R}(G)$ acting multiplicatively on $L^{1}(G) \cap L^{\infty}(G)$.

Lemma A4. The set $\mathscr{G}$ possesses the following properties

(i) let $T, T^{\prime} \in \mathscr{G}$ with $T=U|T|$ be the polar decomposition of $T$, let $\bar{T}=C T C$ : then $T T^{\prime}, T^{*}, \bar{T},|T|, U$ belong to $\mathscr{G}$, and so does $T^{-1}$ if it exists;

(ii) $\mathscr{G} C \mathscr{R}(G)$; thus $T(f * g)=(T f) * g, T \in \mathscr{G}, f \in \mathscr{H}, \Delta^{1 / 2} g \in L^{1}(G)$;

(iii) let $T \in \mathscr{G}$ with $\bar{T}^{*}=T^{n}$. One has

$$
\left\{\begin{array}{l}
f \cdot T^{\wedge} h=T^{\wedge}\{(T f) \cdot h\} \\
(T f) \cdot h=T\left\{f \cdot\left(T^{\wedge} h\right)\right\}
\end{array}, \quad f, h \in L^{2}(G) \cap L^{\infty}(G)\right.
$$

thus

$$
f \cdot T^{\wedge} h=T^{\wedge} T\left\{f \cdot T^{\wedge} h\right\}, \quad f, h \in L^{2}(G) \cap L^{\infty}(G) ;
$$

(iv) each self adjoint projection in $\mathscr{G}$ coincides with $\mathbb{1}$;

(v) $\mathscr{G}$ is a group of unitaries in $\mathscr{R}(G)$ commuting with $C$;

(vi) each $U \in \mathscr{G}$ leaves invariant the * subalgebra $L^{2}(G) \cap L^{\infty}(G)$ of $L^{\infty}(G)$ on which it acts multiplicatively:

$$
U(f \cdot g)=U f \cdot U g, \quad f, g \in L^{2}(G) \cap L^{\infty}(G) .
$$

Proof. (i): the facts that $T T^{\prime}, T^{*}, \bar{T}$, and $T^{-1}$ if it exists, belong to $\mathscr{G}$, are immediately obvious from (A2). Let $T=U|T|$ be the polar decomposition of $T \in \mathscr{G}$, since we have

$$
\begin{aligned}
W^{*}(U|T| \otimes \mathbb{1}) W & =W^{*}(U \otimes \mathbb{1}) W \cdot W^{*}(|T| \otimes \mathbb{1}) W \\
& =U|T| \otimes U|T|=(U \otimes U)(|T| \otimes|T|),
\end{aligned}
$$

8 In the appendix $\lambda_{s}^{\prime}$ is defined in a way which differs from that of the main text 
with equality of the right supports of $W^{*}(U \otimes \mathbb{1}) W$ and $W^{*}(|T| \otimes \mathbb{1}) W$, resp. $U \otimes U$ and $|T| \otimes|T|$, the uniqueness of polar decomposition implies that $|T|, U$ satisfy (A2): since they do not vanish, they belong to $\mathscr{G}$.

(ii): we have, for $T \in \mathscr{G}$ and $s \in G$

$$
W^{*}(T \otimes \mathbb{1})\left(\mathbb{1} \otimes \lambda_{s}^{\prime}\right) W=W^{*}\left(\mathbb{1} \otimes \lambda_{s}^{\prime}\right)(T \otimes \mathbb{1}) W
$$

hence, by (A2) and the fact that $W \in m\left(L^{\infty}(G)\right) \bar{\otimes} \mathscr{R}(G)$,

$$
(T \otimes T)\left(\mathbb{1} \otimes \lambda_{s}^{\prime}\right)=\left(\mathbb{1} \otimes \lambda_{s}^{\prime}\right)(T \otimes T),
$$

i.e. $T \otimes\left(T \lambda_{s}^{\prime}-\lambda_{s}^{\prime} T\right)=0$ whence $T \lambda_{s}^{\prime}=\lambda_{s}^{\prime} T$ since $T \neq 0$.

(iii): we have, for $f, h \in L^{2}(G) \cap L^{\infty}(G), g, k \in \mathscr{H}$, using (A3) and (ii)

$$
\begin{aligned}
& ((T \otimes \mathbb{1}) W(f \otimes g) \mid \bar{h} \otimes k)=\left(W(f \otimes g) \mid T^{*} \bar{h} \otimes k\right)=\left(f \cdot \overline{T^{*}} \overline{\bar{h}} \mid \bar{g} * k^{\eta}\right) \\
& =\left(f \cdot T^{\wedge} h \mid \bar{g} * k^{2}\right)=(W(T f \otimes T g) \mid \bar{h} \otimes k)=\left(T f \cdot h \mid \overline{T g} * k^{2}\right) \\
& =(T f \cdot h \mid \overline{T g * \bar{k}})=\left(T f \cdot h \mid \bar{T}\left(\bar{g} * k^{\jmath}\right)\right)=\left(T^{\wedge}(T f \cdot h) \mid \bar{g} * k^{\nu}\right)
\end{aligned}
$$

whence the first equation (A10). The second follows exchanging $T$ with $T^{n}$ and $f$ with $h$; and the combination of both yields (A11).

(iv): let $E$ be a self-adjoint projection in $\mathscr{G}$ : applying (A11) to $E^{\wedge}$ we have

$$
m_{f} E h=E E^{\hat{\lambda}} m_{f} E h, \quad f, h \in L^{2}(G) \cap L^{\infty}(G) .
$$

Thus $m_{f} E h=E m_{f} E h$, whence, by density, $m_{f} E=E m_{f}$ and $E \in m\left(L^{\infty}(G)\right)^{\prime}$ $=m\left(L^{\infty}(G)\right)$. Thus, by Lemma A3, $E \otimes \mathbb{1}$ commutes with $W$, hence (A2) with $T=E$ yields

$$
W^{*}(E \otimes \mathbb{1}) W=E \otimes \mathbb{1}=E \otimes E .
$$

Thus $E \otimes(E-\mathbb{1})=0$, whence $E=\mathbb{1}$ since $E \neq 0$.

(v) let $T \in \mathscr{G}$ have the polar decomposition $U|T|$, so that $U,|T| \in \mathscr{G}$. By (iv) $U^{*} U$ $=U U^{*}=\mathbb{1}, U$ is unitary. Thus, $T^{*}$, and $T^{*}$, have dense range, thus (A11) implies that

$$
\mathbb{1}=T^{\wedge} T=|T|^{\wedge} U^{\wedge} U|T|
$$

hence that $\||T|\| \geqq 1$. Analogously $|T|^{-1} \leqq 1$. Since $|T| \geqq 0$ we must have $|T|=\mathbb{1}$ : we proved that $T=U$ is unitary. Now from $U^{\wedge}=U^{-1}$, but $U^{\wedge}=C U^{*} C=C U^{-1} C$, thus $U$ commutes with $C$.

(vi): from (A10), we have, for $f, h \in L^{2}(G) \cap L^{\infty}(G)$, since $U$ is unitary,

$$
\|(U f) \cdot h\|_{2} \leqq\|f\|_{\infty} \cdot\|h\|_{2} .
$$

Thus $\left\|m_{U f}\right\|=\|U f\|_{\infty} \leqq\|f\|_{\infty}$ and $U$ leaves $L^{2}(G) \cap L^{\infty}(G)$ invariant. Furthermore since, as we saw in (v), $U^{\wedge}=U^{-1}$, the first equation (A10) with $g=T h \in L^{2}(G) \cap L^{\infty}(G)$ yields (A12).

Proof of Theorem A1. We now endow the subgroup $\mathscr{G}$ of the unitary group of $\mathscr{R}(G)$ with the topology inherited from the weak-*-topology of the latter. $\mathscr{G}$ is then locally compact since it was defined as $\overline{\mathscr{G}} \backslash\{0\}, \overline{\mathscr{G}}$ the closed subset of the weak-*compact unit ball of $\mathscr{B}(\mathscr{H})$ singled out by requiring (A2). $\mathscr{G}$ is furthermore a topological group under this locally compact topology as readily follows from the 
following facts: on the unit ball of $\mathscr{R}(G)$, the weak-*-topology of the latter coincides with the weak-operator topology, which coincides in turn on unitaries with the strong-operator topology due to the fact that

$$
\left(U-U_{0}\right) *\left(U-U_{0}\right)=\left(U_{0}^{*}-U^{*}\right) U_{0}+U_{0}^{*}\left(U-U_{0}\right)
$$

for $U$ and $U_{0}$ unitary. Now the strong operator topology makes $\mathscr{G}$ a topological group, since, for $U, V, U_{0}, V_{0} \in \mathscr{G}$ and $\xi \in \mathscr{H}$

$$
\begin{gathered}
\left\|\left(U V-U_{0} V_{0}\right) \xi\right\| \leqq\left\|\left(V-V_{0}\right) \xi\right\|+\left\|\left(U-U_{0}\right) V_{0} \xi\right\| \\
\left\|\left(U^{-1}-U_{0}^{-1}\right) \xi\right\|=\left\|\left(U_{0}-U\right) U_{0}^{*} \xi\right\| .
\end{gathered}
$$

We thus reached a situation where $\mathscr{G}$ is a locally compact group, with $\lambda: G \rightarrow \mathscr{G}$ a continuous group monomorphism: we now want to show that $\lambda(G)=\mathscr{G}$. For this we use as a technical device the following construction of the Haar measure of $\mathscr{G}$ in terms of $A(G)$. Since $\mathscr{G} \subset \mathscr{R}(G)$ we can embed $A(G)$ as follows into $\mathscr{C}_{\infty}(\mathscr{G})$ : if we set

$$
a(U)=\langle a, U\rangle, \quad a \in A(G), \quad U \in \mathscr{G},
$$

a appears as the restriction to $\mathscr{G}$ of a continuous function on $\overline{\mathscr{G}}$ vanishing at infinity. Now fix $\xi, \eta \in \mathscr{H}$ so that $\bar{\xi} \cdot \eta \in L^{1}(G)$ : by the Riesz theorem there is a Radon measure $\mu_{\eta \xi}$ on $\mathscr{G}$ such that

$$
\langle a, \lambda(\bar{\eta} \cdot \xi)\rangle=\int_{\mathscr{G}} a(U) d \mu_{\eta \xi}(U)=(\eta \mid m(a) \xi),
$$

where the last equality follows from the fact that, if $a=\mid g)(f \mid$ with $f, g \in \mathscr{H}$ the above expression equals

$$
\left(f \mid \lambda(\bar{\eta} \cdot \xi) g^{y}\right)=\left(\eta \mid m\left(\bar{f} * g^{\eta}\right) \xi\right)
$$

as easily checked using (A11). Now we have the property

$$
d \mu_{(\varphi \cdot \eta)(\psi \cdot \xi)}=\bar{\varphi} \cdot \psi d \mu_{\eta \xi}=\bar{\eta} \xi d \mu_{\varphi \psi}, \quad \eta, \xi, \varphi, \psi \in \mathscr{H} \cap A(G),
$$

indeed, in that case, for $a \in A(G)$

$$
(\varphi \cdot \eta \mid m(a) \psi \cdot \xi)=(\varphi \mid m(\bar{\eta} \cdot \psi \cdot a) \xi)=(\eta \mid m(\bar{\varphi} \cdot \psi \cdot a) \xi) .
$$

Using (A26) in the form $|\varphi|^{2} d \mu_{\eta \xi}=\bar{\eta} \xi d \mu_{\varphi \varphi}$ we see that, since the support of $\mu_{\varphi \varphi}$ is included in that of $\varphi$, we can define

$$
d \mu=\frac{1}{|\varphi|^{2}} d \mu_{\varphi \varphi}, \quad \varphi \in \mathscr{H} \cap A(G),
$$

thus obtaining a Radom measure on $\mathscr{G}$ fulfilling

$$
d \mu_{\eta \xi}=\bar{\eta} \cdot \xi d \mu, \quad \eta, \xi \in \mathscr{H} \cap A(G),
$$

i.e.

$$
\begin{gathered}
\int_{G} \bar{\eta}(s) \xi(s) a(s) d s=\int_{\mathscr{G}} \bar{\eta}(U) \xi(U) a(U) d \mu(U)=(\eta \mid m(a) \xi), \\
a \in A(G), \quad \eta, \xi \in \mathscr{H} \cap A(G) .
\end{gathered}
$$


We now show that $\mu$ is a left Haar measure of $\mathscr{G}$. We have indeed, for $U_{0} \in \mathscr{G}$, using the allowable limiting case $a=\mathbb{1}$ in (A30) and taking account of Lemma A4, (v), (vi):

$$
\begin{aligned}
\int \bar{\eta}\left(U_{0}^{-1} U\right) \xi\left(U_{0}^{-1} U\right) d \mu(U) & =\int\left\langle\bar{\eta} \cdot \xi, U_{0}^{*} U\right\rangle d \mu(U) \\
& =\int\left\langle U_{0}(\bar{\eta} \cdot \xi), U\right\rangle d \mu(U) \\
& =\int\left\langle\left(U_{0} \bar{\eta}\right) \cdot\left(U_{0} \xi\right), U\right\rangle d \mu(U) \\
& =\int\left(\overline{U_{0} \eta}\right)(U) \cdot\left(U_{0} \xi\right)(U) d \mu(U) \\
& =\left(U_{0} \eta \mid U_{0} \xi\right)=(\eta, \xi) .
\end{aligned}
$$

Since $\mu$ is a left Haar measure of $\mathscr{G}$, thus has support the whole of $\mathscr{G}$, (A30) implies that the sup norms on $G$ and $\mathscr{G}$ coincide on all $g \in \mathscr{H} \cap A(G)$. Each $U \in \mathscr{G}$ is therefore, according to (A12), a multiplicative functional on $\mathscr{H} \cap A(G)$ continuous for the sup norm on $G$ : by density this functional extends to a continuous character of $\mathscr{C}_{\infty}(G)$ : we thus have a group isomorphism $s: \mathscr{G} \rightarrow G$ such that

$$
U=\lambda(s(U)), \quad U \in \mathscr{G} .
$$

Theorem (A1) can be extended as follows to the case of closed densely defined operators $T$.

Theorem A5. If $T$ is a densely defined closed operator on $\mathscr{H}=L^{2}(G)$ such that

$$
W^{*}(T \bar{\otimes} \mathbb{1}) W=T \bar{\otimes} T,
$$

where $T \bar{\otimes} \mathbb{1}$ and $T \bar{\otimes} T$ mean the closure of the algebraic tensor products, then, with

$$
T=U|T|
$$

the polar decomposition of $T$, there exists an element $s \in G$ and a one parameter subgroup $\{g(t)\}$ of $G$ such that

$$
\left\{\begin{array}{l}
U=\lambda_{s} ; \\
|T|^{i t}=\lambda_{g(t)}, \quad t \in \mathbb{R} .
\end{array}\right.
$$

Proof. Since one has

$$
(T \bar{\otimes} T)^{*}=T^{*} \bar{\otimes} T^{*},
$$

we get as follows the polar decomposition of $T \bar{\otimes} T$ :

$$
T \bar{\otimes} T=(U \otimes U)(|T| \bar{\otimes}|T|) .
$$

The uniqueness of the polar decomposition yields

$$
\left\{\begin{array}{l}
W^{*}(U \otimes \mathbb{1}) W=U \otimes U \\
W^{*}(|T| \otimes \overline{1}) W=|T| \otimes|T| T \mid
\end{array}\right.
$$

By Theorem A1, $U$ must be of the form: $U=\lambda_{s}$ for some $s \in G$. Hence $|T|$ is nonsingular, so that $|T|^{i t}$ yields a one parameter unitary group on $\mathscr{H}$. Since $W$ 
intertwines the functional calculus of $|T| \bar{\otimes} \mathbb{1}$ and $|T| \otimes|T|$, we get

$$
W^{*}\left(|T|^{i t} \otimes \mathbb{1}\right) W=|T|^{i t} \otimes|T|^{i t}, \quad t \in \mathbb{R} .
$$

Hence our assertion follows from Theorem A1.

An element $f \in L^{2}(G)$ is called left bounded if the left convolution: $\xi \in L^{2}(G) \mapsto f * \xi L^{2}(G)$ is bounded, and the corresponding operator on $L^{2}(G)$ is denoted by $\lambda(f)$. It is easy to see that $\lambda(f)$ belongs to $\mathscr{R}(G)$.

Lemma A6. If $\varphi \in A(G)$ and $f \in L^{2}(G)$ is left bounded, then $m_{\varphi} f$ is left bounded and

$$
\left(m_{\varphi} f\right)=\varphi \cdot \lambda(f)
$$

where $\varphi \cdot \lambda(f)$ is defined by (20) and based on the duality between $A(G)$ and $\mathscr{R}(G)$.

Proof. Let $\varphi=\mid \eta)\left(\xi \mid\right.$. By $(20)$, we have $\varphi(s)=\bar{\xi}_{*} \eta^{\llcorner}(s)$. Now we simply compute:

$$
\begin{aligned}
\left(\xi_{1} \mid[\varphi \cdot \lambda(f)] \eta_{1}\right) & \left.=\left\langle W^{*}(\lambda(f) \otimes \mathbb{1}) W, \varphi \otimes\right| \eta_{1}\right)\left(\xi_{1}|\rangle\right. \\
& \left.=\left\langle W^{*}(\lambda(f) \otimes \mathbb{1}) W,\right| \eta\right)\left(\xi|\otimes| \eta_{1}\right)\left(\xi_{1}|\rangle\right. \\
& =\left(\xi \otimes \xi_{1} \mid W^{*}(\lambda(f) \otimes \mathbb{1}) W\left(\eta \otimes \eta_{1}\right)\right) \\
& =\iiint f(r) \eta\left(r^{-1} s\right) \eta_{1}\left(r^{-1} s t\right) \overline{\xi(s)} \overline{\xi_{1}(s t)} d r d s d t \\
& =\iiint f(r) \eta\left(r^{-1} s\right) \eta_{1}\left(r^{-1} t\right) \overline{\xi(s)} \overline{\xi_{1}(t)} d r d s d t \\
& =\iint(\bar{\xi} * \eta)\left(r^{\dagger}\right) f\left(r^{\prime}\right) \eta_{1}\left(r^{-1} t\right) \overline{\xi_{1}(t)} d r d t \\
& =\left\langle\bar{\xi}_{1},(\varphi f) * \eta_{1}\right\rangle .
\end{aligned}
$$

Therefore, $\varphi f$ is left bounded and (A40) follows.

\section{References}

1. Kastler, D., Takesaki, M.: Group duality and the Kubo-Martin Schwinger condition. Commun. Math. Phys. 70, 193-212 (1979)

2. Takesaki, M.: Noncommutative group duality and the Kubo-Martin Schwinger condition. In: Quantum fields - algebras, processes, L. Streit (ed.), Proc. Bielefeld Encounters in Phys. and Math. Berlin, Heidelberg, New York: Springer 1980. 197-205

3. Araki, H., Haag, R., Kastler, D., Takesaki, M.: Extension of states and chemical potential. Commun. Math. Phys. 53, 97-134 (1977)

4. Haag, R., Kastler, D., Trych-Pohlmeyer, E.: Stability and equilibrium. Commun. Math. Phys. 38, 173-193 (1974)

5. Bratteli, O., Kishimoto, A., Robinson, D.W.: Stability properties and the KMS-condition. Commun. Math. Phys. 61, 209-238 (1978)

6. Eymard, P.: L'algebra de Fourier d'un groupe localement compact. Bull. Soc. Math. Fr. 92, 181236 (1964)

7. Stinespring, W.F.: Integration theorems for gages and duality for unimodular groups. Trans. Am. Math. Soc. 90, 15-56 (1959)

8. Saitô, K.: On a duality for locally compact groups. Tôhoku Math. J. 20, 355-367 (1968)

9. Tatsuuma, N.: A duality theorem for locally compact groups. J. Math. Kyoto Univ. 6, 187-293 (1967)

10. Tatsuuma, N.: An extension of AKHT theory of locally compact groups. Kokyuroku, RIMS 314, 88 (1977) 
11. Ciorănescu, I., Zsidó, L. : Analytic generators of one-parameter groups. Tôhoku Math. J. 28, 327362 (1976)

12. Takesaki, M.: Theory of operator algebras. I. Berlin, Heidelberg, New York: Springer 1979

13. Ioanescu-Tulcea, A.C.: On the existence of a lifting commuting with the left translations of an arbitrary locally compact group. Proc. Fifth Berkeley Symposium on Math. Sat. and Prob; (1967) pp. 63-67

14. Pusz, W., Woronowicz, S.L.: Passive states and KMS states for general quantum systems. Commun. Math. Phys. 58, 273 (1978)

15. Araki, H., Kishimoto, A.: Symmetry and equilibrium states. Commun. Math. Phys. 52, 211 (1977)

Communicated by R. Jost

Received October 28, 1981 